\title{
Intuitionistic fuzzy system based latent fingerprint enhancement and matching using minutiae and SIFT feature
}

\author{
Adhiyaman Manickam and Ezhilmaran Devarasan \\ Department of Mathematics, School of Advanced Science, VIT University - Vellore, India \\ e-mails: adhimsc2013@gmail.com, ezhil.devarasan@yahoo.com
}

Received: 8 October 2017 Revised: 3 December $2017 \quad$ Accepted: 4 December 2017

\begin{abstract}
In this work, we developed a model for latent fingerprint enhancement and matching algorithm, which requires manually marked ROI latent fingerprints. The proposed model includes two phases (i) Latent fingerprints contrast enhancement using type-2 intuitionistic fuzzy set (ii) Extract the minutiae and Scale Invariant Feature Transformation (SIFT) features from the latent fingerprint image. For matching, this algorithm has been figured based on minutiae and SIFTS points which inspect $\mathrm{n}$ number of images and the scores are calculated by Euclidean distance. We tested our algorithm for matching, using some public domain fingerprint databases such as FVC2004 and IIIT-latent fingerprint which indicates that by fusing the proposed enhancement algorithm, the matching precision has fundamentally moved forward.
\end{abstract}

Keywords: Latent fingerprint image, Type-2 intuitionistic fuzzy set, Feature extraction, Enhancement, Matching.

2010 Mathematics Subject Classification: 94Dxx, 03E72, 03B52, 93C42.

\section{Introduction}

Physical attributes and behavior are the parameters for identifying a person in biometric security. The biometric traits, for example, face, gait, ear, odor fingerprint, hand geometry, iris, retina, hand vein, facial thermogram, signature, voice print, and keystroke dynamics which are exceptionally suitable for human acknowledgment because of their singularity, integrality and consistency [10]. Among all biometric traits, fingerprints have the elevated amount of dependability and widely used by criminological specialists as a part of criminal examinations. Typically, latent fingerprints are low in quality (Figure 1). It is necessary to enhance the original image to acquire a better quality. The major idea of image enhancement is to create 
another image in such a way that, it uncovers data for investigation more than the first image. In this current investigation, the fingerprint image contrast enhancement is carried out by using type-2 intuitionistic fuzzy set and the algorithm is performing for matching of manually marked Region of Interest (ROI) latent fingerprints.
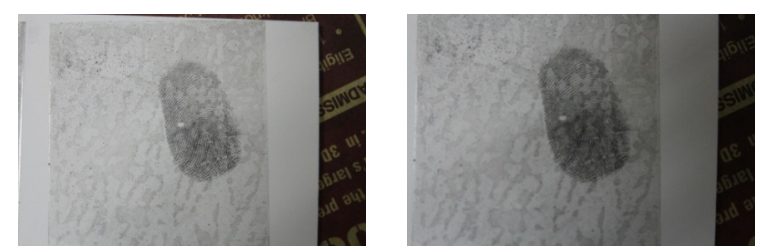

Figure 1. Example of latent fingerprint images

(Database of Indraprastha Institute of Information Technology (IIIT), Delhi)

The rest of this paper is composed as follows: Section 2 gives a short narrative of background study of this work. Section 3 explains the notion of type-2 intuitionistic fuzzy set theory. Section 4 describes proposed methodology include contrast enhancement and minutiae and SIFT feature based matching for latent fingerprints. Section 5 displays the results and discussion and to finish the conclusion in Section 6.

\section{Related works}

The fingerprint image enhancement is carried by Mao et al. [11] with the help of Gabor filter. The integration of Anisotropic Filter (AF) and Directional Median Filter (DMF) techniques are used for fingerprint image enhancement which is discussed by Wu et al. (2004) [15]. Jayaram et al. [6] proposed a fuzzy inference system based contrast enhancement of gray level images. Selvi et al. [14] described the fuzzy based fingerprints enhancement method which is used to reduce the noise free image from the original image for further processing. Chaira [3] used the soft computing technique such as type-2 fuzzy set for medical and fingerprint image enhancement. Chaira and Ray [4] disclosed a novel technique to create fuzzy edges in medical images using type-2 fuzzy set theory. It has taking into account the calculation of minimum and maximum values of the intensity levels of the image in a $3 \times 3$-pixel neighborhood to form two image matrices with maximum and minimum values.

Yoon et al. [18] proposed an algorithm for latent fingerprint image enhancement which only requires minimum margin in ROI and singular points to promote the automatic matching accuracy. Later, they have proposed a latent fingerprint enhancement algorithm, which expects manually marked ROI and singular points [17]. Further in 2013, they have introduced the algorithm for Fingerprint Image Quality (LFIQ), which is used to latent fingerprint exemplar and crime investigation [19].

Malathi and Meena [9] have demonstrated a method for partial fingerprint matching based on score level fusion by using pore and SIFT features. Park et al. [12] have discussed a new method for fingerprint verification using SIFT feature. It extracts the SIFT feature points in scale space and perform matching based on the texture information around the feature points using the SIFT operator. Yang et al. [16] explained an algorithm for fingerprint matching using SIFT points which is used to eliminate the superfluous error matching points to improve 
matching accuracy. Lowe [8] explores the effectiveness of the SIFT for image matching which is a popularly used interest point detection method often applied to image matching the Harris corner detector.

\section{Preliminaries of fuzzy set}

In 1965, Zadeh defined the notion of fuzzy set theory. It deals with degree of membership function. Later in 1983, Atanassov introduced the intuitionistic fuzzy set theory which deals with degree of membership and non-membership functions. It has a vital role in image processing, for example, image enhancement, morphological image, edge detection and so on. Mathematically, the definitions as follows,

Definition 3.1 (Zadeh, 1965) [20] Typically, the fuzzy set theory indicates the membership functions which contain the closed interval range $[0,1]$. The degree of membership function denoted as a fuzzy set A by $\mu_{A}$. It can be represented as follows $A=\left\{\left\langle x, \mu_{A}(x)\right\rangle \mid x \in X\right\}$ where $\mu_{A}(x): X \rightarrow[0,1]$.

Definition 3.2 (Atanassov, 1986) [1] Intuitionistic fuzzy set theory was introduced by Atanassov which includes two functions such as degree of membership and non-membership functions respectively. The degree of non-membership function denoted as an intuitionistic fuzzy set $\mathrm{A}$ by $v_{A}$. It can be mathematically represented as follows

$A=\left\{\left\langle x, \mu_{A}(x), v_{A}(x)\right\rangle \mid x \in X\right\}$; where $\mu_{A}(x), v_{A}(x): X \rightarrow[0,1] . \pi_{A}(x)=1-\mu_{A}(x)-v_{A}(x)$ is the representation of the third parameter which is called the hesitation degree.

Definition 3.3 Type-2 fuzzy set is helpful in circumstance where the membership values are such that it is hard to concur with accurate membership values. This is due to the way that some uncertainty might exhibit fit as a fiddle or different parameters. Type-2 fuzzy set accounts this uncertainty by considering another level of opportunity for better representation of instability where the membership functions are themselves fuzzy.

Type-2 fuzzy set considers some more uncertainty in the form of membership function [7]. It can be written as follows: $A_{\text {Type-2 }}=\left\{\left\langle x, \widehat{\mu}_{A}(x)\right\rangle \mid x \in X\right\}$, where $\widehat{\mu}_{A}(x)$ is the type-2 membership function.

\section{Proposed methodology}

In this section, we investigate the latent fingerprints image Pre-processing, Contrast enhancement, Minutiae and SIFT feature extraction and Matching.

\subsection{Pre-Processing}

The pre-processing is categories into two processes which are Binarization and Thinning. The aim of pre-processing is a change of the image that upgrades a few elements important for 
further handling. Gray scale image is reformed into binary image (Figure 2 (a)) which is labeled as binarization. This means each pixel is stored as a single bit, i.e., 0 or 1 . The usability of image thinning (Figure 2 (b)) is reducing the darkness of ridge lines. It is used in matching process because better image quality is required. Thinning is the transformation of a digital image into a simplified image but does not change over the original $(x, y)$ area. Ridge thinning is utilized to destruct the additional pixel of ridges until just one pixel broad [5].
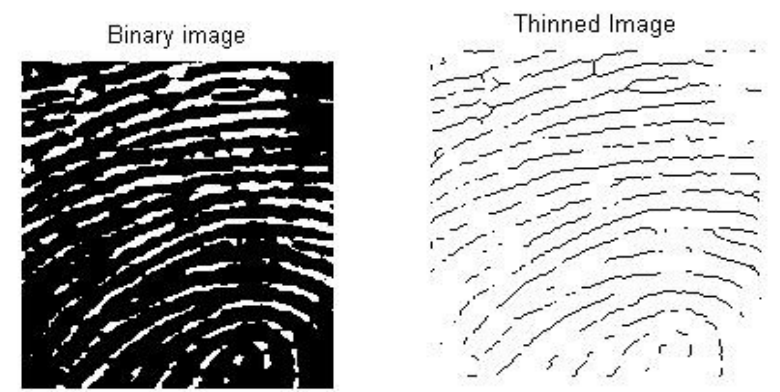

Figure 2. (a) Binary image (b) Thinned image

\subsection{Contrast enhancement using type-2 intuitionistic fuzzy set}

This enhancement method is based on window scheme. The original image is split into six windows and each window maintained the same size for further processing. Enhancement is obtained from each window.

The following formula is used to fuzzified the $N \times N$ image:

$$
\mu_{A}^{f u z}\left(g_{i j}\right)=\frac{g_{i j}-g_{\min }}{g_{\max }-g_{\min }},
$$

where $g$ is the gray level of the image ranges from 0 to L-1. $g_{\max }$ and $g_{\min }$ are the maximum and minimum values of the image.

The membership and non-membership function is calculated for each window using Takagi-Sugeno-Kang (TSK) type intuitionistic fuzzy set (IFS) generator.

The TSK type IFS generator is followed as [2]:

$$
K(\mu(g))=\frac{(1-\mu(g))}{(1+\lambda \mu(g))}, \lambda>0,
$$

where $K(1)=0, K(0)=1$. The non-membership function is derived by using Sugeno type intuitionistic fuzzy generator

$$
v_{A}^{w i n}\left(g_{i j}\right)=\frac{1-\mu_{A}^{f u z}\left(g_{i j}\right)}{1+\lambda \mu_{A}^{f u z}\left(g_{i j}\right)} .
$$

The TSK-IFS become as:

$$
A_{\lambda}^{I F S}=\left\{\left\langle x, \mu_{A}\left(g_{i j}\right), \frac{1-\mu_{A}\left(g_{i j}\right)}{1+\lambda \cdot \mu_{A}\left(g_{i j}\right)}\right\rangle \mid g_{i j} \in A\right\} .
$$

For all windows, the hesitation degree can be mathematically written as follows: 


$$
\pi_{A}\left(g_{i j}\right)=1-\mu_{A}^{f u z}\left(g_{i j}\right)-v_{A}^{w i n}\left(g_{i j}\right) .
$$

In this experiment $\lambda=1$ is utilized to maintain the quality of the image because on raising the $\lambda$ value, the enhanced image will be worsening. At the same time, as $\lambda$ increases, the Sugeno generator will decrease thereby the non-membership value will decrease and the hesitation degree will increase.

The average value of the enhanced features is considered in each window. As has been discussed in Section 3, due to hesitation degree, membership values lie in an interval range, so, the modified membership value is written as:

$$
\mu_{A}^{\bmod }\left(g_{i j}\right)=\mu_{A}^{f u z}\left(g_{i j}\right)-\text { mean_window* } \pi_{A}\left(g_{i j}\right) .
$$

Here, we introduce the type-2 fuzzy membership function with based on definition 3.3 in section 3. Based on the image quality the value of $\alpha$ is obtained by using trial and error method.

The Hamacher T-conorm is used to develop the new membership function that considers both the upper and lower functions [7]:

$$
\mu^{\text {type-2}}\left(g_{i j}\right)=\frac{\mu^{\text {upper }}+\mu^{\text {lower }}+(\lambda-2) \mu^{\text {upper }} \cdot \mu^{\text {lower }}}{1-(1-\lambda) \mu^{\text {upper }} \mu^{\text {lower }}}
$$

$\mu^{u p p e r}\left(g_{i j}\right)$ and $\mu^{\text {lower }}\left(g_{i j}\right)$ are the upper and lower membership function of the type-2 fuzzy set respectively which are computed using Definition 3.

$$
\mu^{u p p e r}\left(g_{i j}\right)=\left[\mu_{A}^{f u z}\left(g_{i j}\right)\right]^{\alpha}, \quad \mu^{\text {lower }}\left(g_{i j}\right)=\left[\mu_{A}^{f u z}\left(g_{i j}\right)\right]^{1 / \alpha}
$$

In this case of $\lambda$ is taken as $\lambda=10 \times i m_{-} a v g$, where $i m_{-} a v g$ is the average value of the pixels in the image. Form this experiment results, the value ' 10 ' would be the better and results are achieved by separating the images into several windows.

\subsection{Minutiae extraction}

In this work, the minutiae are extracted by using Crossing Number $(\mathrm{CN})$ for matching. This technique extracts the termination and bifurcations from the skeleton image by examining at the nearby neighborhood of every ridge pixel utilizing a $3 \times 3$ window.

The ridge pixel $P$ is calculated by using $\mathrm{CN}$. It can be mathematically written as follows [13],

$$
C . N=0.5 \sum_{I=1}^{8}\left|P_{i}-P_{i+1}\right|, P_{9}=P_{1},
$$

where $P_{i}$ represent the pixel value in the neighborhoods of $P$. The eight neighborhoods are scanned in an anti-clockwise direction for a pixel $P$. The ridge pixels are computed and classified based on its $\mathrm{CN}$ value.

\begin{tabular}{|l|l|l|}
\hline$P_{4}$ & $P_{3}$ & $P_{2}$ \\
\hline$P_{5}$ & $P_{4}$ & $P_{1}$ \\
\hline$P_{6}$ & $P_{7}$ & $P_{8}$ \\
\hline
\end{tabular}


Figure 3 shows the ridge pixel with $\mathrm{CN}$ value. $\mathrm{CN}$ value one and three represents the ridge ending point and bifurcation points respectively.

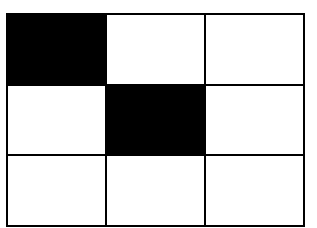

(a)

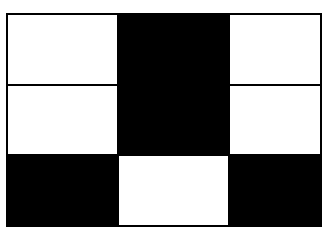

(b)

Figure 3. (a) Ridge ending and (b) Bifurcation pixel

The following steps are following for extracted the minutiae,

- The coordinates are $x$ and $y$,

- Types of minutiae are instructed and Orientation of the associated ridge segment.

All the minutiae points are situated at a particular place in fingerprint image and it is put away as information (Figure 4). The location of every point in the digital image is given by the pixel position. Ridge ending and bifurcation points are easily taken and stored independently. This algorithm is compared to the computed values with stored minutiae values by calculating the Euclidean distance between the query image and image database.

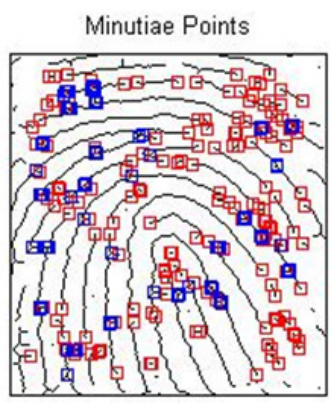

Figure 4. Minutiae points in latent fingerprint image

\subsection{SIFT feature extraction}

SIFT was initially developed for general purpose of object recognition. SIFT method extracts distinctive invariant features from images, which perform consistent matching between different views of an object or scene. Image content is transformed into local feature coordinates that are invariant to translation, rotation, scale, and other imaging parameters (Figure 5). There are four major stages of computation used for generating the SIFT features such as scale-space extrema detection, key point localization, orientation assignment and the key point descriptor. These major stages are performed as follows: pick the key locations at local maximum and minimum of a Difference of Gaussian (DOG) function applied in scale space, which is constructed by successively down sampling the input image. Maximum and minimum of this scale space function are decided by comparing each pixel to its neighbors. 


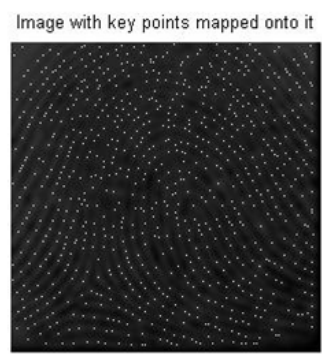

Figure 5. SIFT points of latent fingerprint image

\subsection{Matching for latent fingerprint image}

The algorithm is performed as follows (Figure 6):

Step 1: Introduce the input latent fingerprint images

Step 2: Acquire the input image

Step 3: Convert the Gray scale image into the binary image and apply the thinning process to the image

Step 4: Latent fingerprint image contrast enhancement using type-2 intuitionistic fuzzy set

Step 5: Extract the fingerprint features from the image

Step 6: Pick up the query image form the database and extract the features for query image

Step 7: Calculate the Euclidean distance between query image and database image.

Step 8: Sort the output image and find out which one is perfect match to the given query image

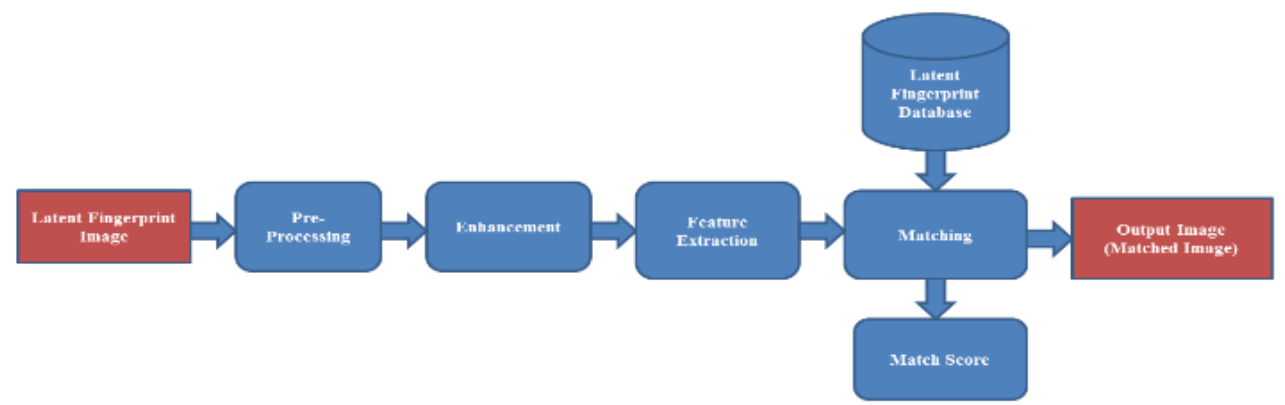

Figure 6. Block diagram of proposed model

Mathematically, the Euclidean distance is the 'ordinary' distance between two points in Euclidean space.

$$
D(p, q)=\sqrt{\sum_{i=1}^{n}\left(p_{i}-q_{i}\right)^{2}} .
$$

After the completion of the matching process, the matched image will be displayed on the screen. 


\section{Experimental results and discussion}

The fingerprint experiments are dealing with two different databases such as Fingerprint Verification Competition-2004 (FVC-2004) and Indraprastha Institute of Information Technology (IIIT) Latent Fingerprint Database. These both are public available fingerprint databases. FVC-2004 consists of four databases and each database image has different size and resolution (Available at http://bias.csr.unibo.it/fvc2004/download.asp.)

In particular, FVC-2004, we are used FVC-2004 database 1. IIIT Latent Fingerprint Database contains 15 subjects (Available at http://www.iab-rubric.org/resources.html). Each subject has all 10 fingerprints. Each image is of $4752 \times 3168$ pixel in size and has been scanned at 500 pixels per inch as a grayscale image. We obtain the unacceptable computation error in latent fingerprint images due to large pixel size. Therefore, the ROI is manually marked (See Figure 7) and it will be changed as a size of $508 \times 661$ for better results. Therefore, the results are obtained based on manually marked ROI image. It is only applicable for latent fingerprint images, but not in fingerprint images. The enhancement and matching algorithm is mainly focuses on latent fingerprint images. In addition, we are tested above mentioned ordinary fingerprint databases (FVC-2004), which are very clear images, compare than latent fingerprints.

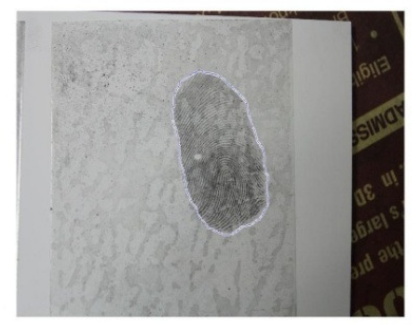

Figure 7. Manually marked ROI of latent fingerprint image

\subsection{Latent fingerprint image contrast enhancement}

The enhancement is carried out by using type-2 intuitionistic fuzzy set. It is used to connect the broken ridge and valley structure for perfect matching. (See Figures 8 and 9, and Table 1).

The latent fingerprint image quality evaluation is very difficult because it contains a lot of uncertainty and poorly illuminated. The evaluation uses the linear index of fuzziness/fuzzy entropy for calculating the fuzziness in enhanced image. The linear index of fuzziness is less than the original image and this is the feature of enhancement. The linear index of fuzziness as follows:

$$
\text { L.I }=\frac{2}{N \times N} \sum \sum \min \left(\mu_{m n}, 1-\mu_{m n}\right)
$$

Table 1. Performance comparison using linear index of fuzziness

\begin{tabular}{|c|c|c|}
\hline Databases & Original image & Proposed type-2 intuitionistic fuzzy set \\
\hline IIIT-latent fingerprint & 0.5162 & 0.2702 \\
\hline FVC-2004 database 1 & 0.4691 & 0.1912 \\
\hline
\end{tabular}




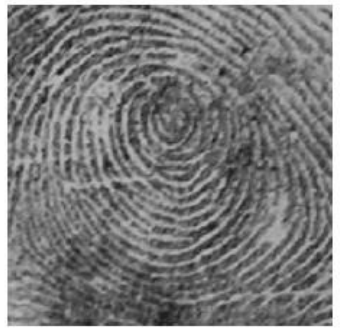

(a)

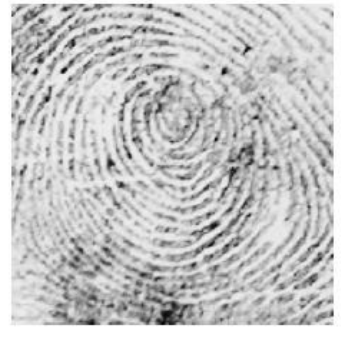

(b)

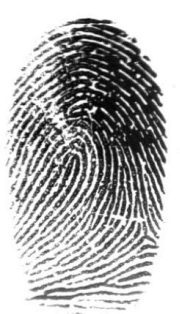

(a)

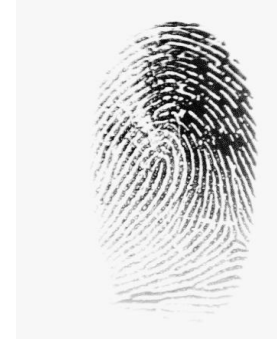

(b)

Figure 8. IIIT latent database:

(a) Original image; (b) Enhancement image

Figure 9. FVC-2004 Database 1:

(a) Original image; (b) Enhancement image

\subsection{Minutiae based latent fingerprints matching}

The latent fingerprint images contain fewer amounts of minutiae points because of un-cleared ridge and valley structure. This noise leads complexity to identify the person. Therefore, the algorithm works on proper pre-processing which is used to identify the exact person. In order to, this algorithm performs to extract and count the minutiae points from the image simultaneously.

Table 2. Minutiae and SIFT match score for FVC- 2004 database 1

\begin{tabular}{|c|c|c|c|c|c|}
\hline $\begin{array}{c}\text { Images } \\
\text { (Tests })\end{array}$ & Size & BEMMS & AEMMS & BESMS & AESMS \\
\hline Test-1 & $640 \times 480(307 \mathrm{~K}$ pixels $)$ & 00.0000 & 00.0000 & 00.0000 & 00.0000 \\
\hline Test-2 & $640 \times 480(307 \mathrm{~K}$ pixels $)$ & 56.8859 & 4.3424 & 11.4961 & 10.3217 \\
\hline Test-3 & $640 \times 480(307 \mathrm{~K}$ pixels $)$ & 67.6831 & 46.8753 & 99.7976 & 90.2341 \\
\hline Test-4 & $640 \times 480(307 \mathrm{~K}$ pixels $)$ & 84.3445 & 72.8975 & 122.0328 & 103.3988 \\
\hline Test-5 & $640 \times 480(307 \mathrm{~K}$ pixels $)$ & 90.0278 & 78.0043 & 143.3901 & 130.7778 \\
\hline Test-6 & $640 \times 480(307 \mathrm{~K}$ pixels $)$ & 134.9244 & 89.9811 & 155.4772 & 145.3212 \\
\hline Test-7 & $640 \times 480(307 \mathrm{~K}$ pixels $)$ & 159.0503 & 92.0912 & 188.8372 & 152.0908 \\
\hline Test- 8 & $640 \times 480(307 \mathrm{~K}$ pixels $)$ & 163.2483 & 107.6532 & 211.1022 & 176.3332 \\
\hline Test-9 & $640 \times 480(307 \mathrm{~K}$ pixels $)$ & 217.8302 & 112.5400 & 218.1120 & 187.0000 \\
\hline Test-10 & $640 \times 480(307 \mathrm{~K}$ pixels $)$ & 220.4223 & 126.2310 & 227.9650 & 198.3432 \\
\hline
\end{tabular}

Table 3. Minutiae and SIFT match score for IIIT latent fingerprint database

\begin{tabular}{|c|c|c|c|c|c|}
\hline $\begin{array}{c}\text { Images } \\
\text { (Tests) }\end{array}$ & Size & BEMMS & AEMMS & BESMS & AESMS \\
\hline Test-1 & $508 \times 661(342 \mathrm{~K}$ pixels) & 00.0000 & 00.0000 & 00.0000 & 00.0000 \\
\hline Test-2 & $508 \times 661(342 \mathrm{~K}$ pixels) & 16.2788 & 06.5647 & 12.8767 & 09.2948 \\
\hline Test-3 & $508 \times 661(342 \mathrm{~K}$ pixels) & 25.4951 & 16.8974 & 38.9902 & 23.3849 \\
\hline Test-4 & $508 \times 661(342 \mathrm{~K}$ pixels) & 46.8188 & 37.3224 & 65.0982 & 29.0483 \\
\hline Test-5 & $508 \times 661(342 \mathrm{~K}$ pixels) & 48.7647 & 39.9875 & 70.8739 & 68.3475 \\
\hline Test-6 & $508 \times 661(342 \mathrm{~K}$ pixels) & 59.7746 & 54.6483 & 87.9837 & 71.9483 \\
\hline Test-7 & $508 \times 661(342 \mathrm{~K}$ pixels) & 74.4648 & 66.9573 & 101.8837 & 79.4837 \\
\hline Test- 8 & $508 \times 661(342 \mathrm{~K}$ pixels $)$ & 85.9069 & 73.9111 & 109.9833 & 97.6837 \\
\hline Test-9 & $508 \times 661(342 \mathrm{~K}$ pixels) & 112.6144 & 87.3433 & 143.9384 & 131.0063 \\
\hline Test-10 & $508 \times 661(342 \mathrm{~K}$ pixels) & 129.1240 & 114.3339 & 164.0394 & 139.3386 \\
\hline
\end{tabular}


Here, BEMMS - Before Enhancement Minutiae Match Score, AEMMS - After Enhancement Minutiae Match Score, BESMS - Before Enhancement SIFT Match Score, AESMS - After Enhancement SIFT Match Score.

Next we find the distance between two minutiae points (database image and query image) by using Euclidean formula. Similarly, the SIFT points are used for the same. Then the matching scores are calculated with the help of the proposed method and it is tested with 10 images from each database but it's applicable for n-number of images. Furthermore, if a query image matches with any one of the template's images, which match score is equal to 0 (exact match). Otherwise similarity matches score will be shown (Tables 2, 3 and Figures 10 and 11).
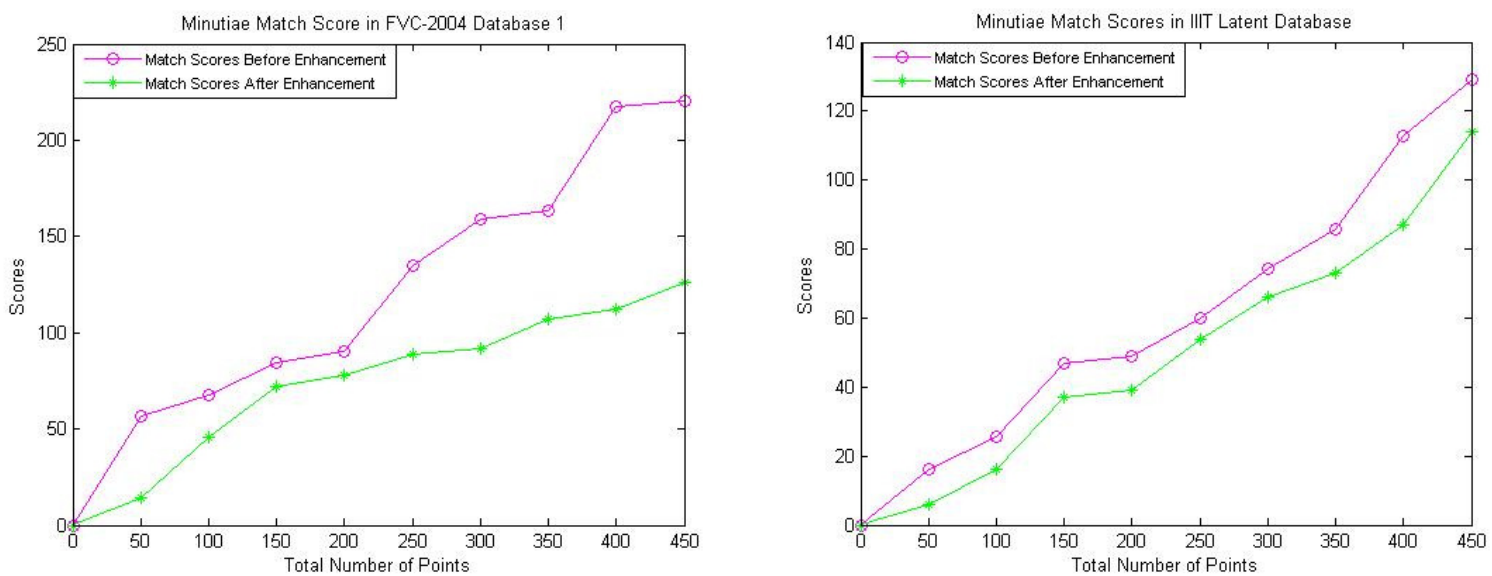

Figure 10. Comparison of before and after enhancement in all databases for minutiae features
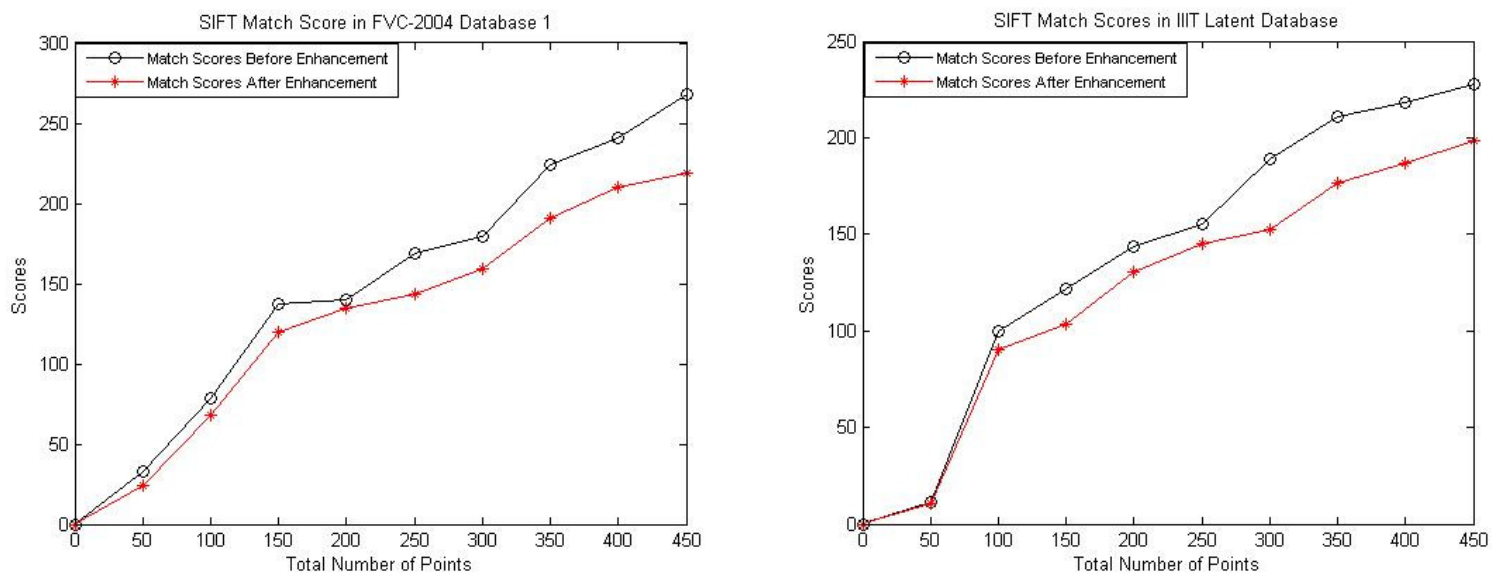

Figure 11. Comparison of before and after enhancement in all databases for SIFT features

\section{Conclusions}

In this work, the latent fingerprints contrast enhancement method is derived using type-2 intuitionistic fuzzy set and matching algorithm is performed based on fingerprint feature points (Minutiae and SIFT) with different databases. From this observation, the overall results indicate that the significance of the enhancement. Therefore, the enhancement step is used to elevate the 
matching accuracy for commercial matcher. This practice helps in shortening the response time of latent fingerprint matching an urgent issue because these latent fingerprints are fully enhanced. We plan to extend the current work along with large public latent fingerprint database by matching and measuring the similarity between the latent fingerprint images. It consider the minutiae feature for reduce the time complexity.

\section{References}

[1] Atanassov, K. T. (1986) Intuitionistic fuzzy sets, Fuzzy sets and Systems, 20(1), 87-96.

[2] Bustince, H., Kacprzyk, J., \& Mohedano, V. (2000) Intuitionistic fuzzy generators application to intuitionistic fuzzy complementation, Fuzzy Sets and Systems, 114, 485504.

[3] Chaira, T. (2013) Contrast enhancement of medical images using Type II fuzzy set, Proc. of the IEEE National Conference on Communications, 1-5.

[4] Chaira, T., \& Ray, A. K. (2014) Construction of fuzzy edge image using interval type II fuzzy set, International Journal of Computational Intelligence Systems, 7, 686-695.

[5] Diefenderfer, G. T. (2006) Fingerprint recognition. DTIC Document, Naval post graduate school, Monterey California.

[6] Jayaram, B., Narayana, K., \& Vetrivel, V. (2011) Fuzzy Inference System based Contrast Enhancement, Proc. of The International Conference on EUSFLAT-LFA, 311-318.

[7] Lee, K. H. (2006) First Course on Fuzzy Theory and Applications, Springer, Science and Business Media, 27.

[8] Lowe, D. G. (2004) Distinctive image features from scale-invariant keypoints, International Journal of Computer Vision, 60, 91-110.

[9] Malathi, S., \& Meena, C. (2011) Improved partial fingerprint matching based on score level fusion using pore and sift features. Proc. of the IEEE International Conference on Process Automation Control and Computing, 1-4.

[10] Maltoni, D., Maio, D., Jain, A. K., \& Prabhakar, S. (2009) Handbook of fingerprint recognition. Springer.

[11] Mao, K., Zhu, Z., \& Jiang, H. (2010) A fast fingerprint image enhancement method, Proc. of the Third IEEE International Joint Conference on Computational Science and Optimization, 222-226.

[12] Park, U., Pankanti, S. , \& Jain, A. K. (2008) Fingerprint verification using SIFT features, Proc. of the International Society for Optics and Photonics in SPIE Defense and Security symposium, 69440K-69440K.

[13] Rutovitz, D. (1966) Pattern recognition, Royal Statistical Society, 129, 504-530. 
[14] Selvi, M., \& George, A. (2013) FBFET: Fuzzy based fingerprint enhancement technique based on adaptive thresholding, Proc. of the IEEE Fourth International Conference on Computing, Communications and Networking Technologies, 1-5.

[15] Wu, C., Shi, Z., \& Govindaraju, V. (2004) Fingerprint image enhancement method using directional median filter, Proc. of the International Society for Optics and Photonics Defense and Security, 66-75.

[16] Yang, Y., Liu, W., \& Zhang, L. (2010) Study on improved scale invariant feature transform matching algorithm, Proc. of the Second Pacific-Asia Conference on Circuits, Communications and System, 1, 398-401.

[17] Yoon, S., Cao, K., Liu, E., \& Jain, A. K. (2013) LFIQ: Latent fingerprint image quality, Proc. of the Sixth IEEE International Conference on Theory, Applications and Systems, $1-8$, Arlington.

[18] Yoon, S., Feng J., \& Jain, A.K. (2011) Latent fingerprint enhancement via robust orientation field estimation, Proc. of the IEEE International Joint Conference on Biometrics, 1-8.

[19] Yoon, S., Liu, E., \& Jain, A. K. (2015) On latent fingerprint image quality, Proc. of the International Workshop on Computational Forensics, 67-82.

[20] Zadeh, L.A. (1965) Fuzzy sets, Information and Control, 8, 338-353. 